\title{
Modeling of variable magnetic elements including hysteresis and Eddy current losses
}

\author{
Sarah Saeed, Jorge García, Ramy Georgious \\ Dept. of Elec., Computer, and System Engineering \\ University of Oviedo \\ Gijón, Spain \\ E-mail: hazkialsarah.uo@uniovi.es, garciajorge@uniovi.es, georgiousramy@uniovi.es.
}

\begin{abstract}
In this paper, a full model to simulate variable magnetic elements in power electronic converters is developed. The derived model is based on the reluctance equivalent circuit of magnetic systems. Specifically, the model considers core losses, determined through Jiles-Atherton hysteresis model, and Eddy current losses obtained using Dowell empirical formulas. The frequency dependence of the device losses is also considered. The model is compared to experimental measurements in order to verify its validity and accuracy, and then it is applied to study and characterize the double E-core variable inductor structure.
\end{abstract}

Keywords—magnetics modeling; variable inductor; hysteresis; Eddy currents; saturable core.

\section{INTRODUCTION}

Understanding the behavior of a magnetic device is essential to foster the performance of Power Electronic Converter (PEC) based systems. In this context, many models have been developed to characterize the magnetic core material in terms of a relationship between magnetic flux density and field intensity referred to as hysteresis curve. In [1], an initial survey has been conducted classifying the existing models, for different frequencies, bias conditions, and temperatures of interest. It aims to provide comparable information for models and their availability in some circuit simulators. A more recent literature review of magnetic regulators, fundamentals, modeling and design has been comprehensively presented in [2]. From the state of the art study, the options for modeling magnetic devices are confined in three directions: Finite Elements Analysis (FEA), gyrator-capacitor model [3], and reluctance equivalent circuit [4]. As the complexity of magnetic devices increases, the theoretical and empirical models turn too complicated to predict the behavior of the device in a practical and simple manner. On the other hand, incorporating those concepts to a computerbased simulation provides good compromise between convenience, accuracy and numerical efficiency. Consequently, many efforts have been directed towards computer-based simulations, especially time-domain models [5] [6].

Variable magnetic elements allow for additional degrees of freedom in the design and control of PECs. This is particularly useful in resonant converters, where the usual frequency control has some drawbacks due Electro-Magnetic Interference (EMI)

This work has been partially supported by the Spanish Government, under the research grant ENE2016-77919, "Conciliator" Project (Innovation Development and Research Office-MEC). Also the work has been partially supported by the government of the Principality of Asturias, under "Severo Ochoa" program of predoctoral grants for training in research and university teaching, grants number BP16-133, and BP13-138. issues, synchronization, variable sampling time, etc., especially for large range of variation. If variable magnetics are used, the same control margins can be obtained at a constant switching frequency, therefore allowing for an optimization of the EMI filters, and sampling procedures. In other applications such as the Dual Active Bridge (DAB) converter, in addition to adding a new degree of freedom to the control, the inclusion of variable magnetics can increase operation parameters such as the soft switching margins, etc. [7] [8].

In all cases, it is necessary to investigate the overall PEC incorporating the variable magnetic device. The aim of this work is to develop a circuit-based, time-domain model of the variable magnetic element able to work in different platforms (e.g. LTSpice, MATLAB-Simulink and PSIM), with equally valid accuracy. The model includes device losses, mainly core losses, $\mathrm{AC}$ winding losses, and Eddy currents. This enables the use of only one simulator environment for the whole electromagnetic system, with acceptable accuracy in compromise with complication and time required for FEA models.

Section II presents an overview of the variable magnetic device structure that will be used, and the models of interest in literature. Section III explains the model for core losses. This section also provides an idea on the implementation of the model equations, on the validation of the model against experimental measurement, and on the approach to estimate the model parameters as a function of operation frequency. Later, in Section IV, the model of the winding losses is presented, and validated against experimental results. After that, Section V explains the use of the losses model to implement the full device. In Section VI, the proposed simulation model is validated in comparison to the previous models that does not include losses, and experimental results are provided. Finally, Section VII summarizes the conclusions of the work.

\section{Modeling OF VARIABle Magnetic ELEMENTS}

A variable magnetic device can be modeled depending on its operation region on the $\mathrm{BH}$ curve: linear, knee, or nonlinear regions [5]. To attain accurate results, the different involved device losses must be considered: hysteresis, and Eddy current losses (which becomes significant with the increase of frequency of operation), and also winding losses (proximity and skin effect losses). Ready-made modules are available in many circuit simulation environments [9] [10]. 
From the study of the state of the art, the double E-core structure, depicted in Fig. 1, is selected to be the most appropriate and most comprehend in literature [11]. Due to the main winding $\left(N_{c}\right)$, an AC flux $\left(\phi_{C}\right)$ circulates through the center arm and splits to the outer arms. Applying a relatively small DC bias current $\left(I_{b}\right)$ to the bias control windings $\left(N_{b}\right)$, a DC flux $\left(\phi_{R}\right.$ or $\left.\phi_{L}\right)$ is produced which circulates mainly through the outer (ungapped) circumference of the core [11]. This flux can bias the operation of the magnetic material towards nonlinear region, causing the inductance seen from the main winding terminals to vary.

The SPICE-based reluctance equivalent circuit provided by authors in [5] is thus found to be of most interest. Fig. 2 shows the reluctance equivalent circuit for the double E-core variable inductor. The voltage sources in the circuit represent the magnetomotive forces due to the bias control windings $\left(N_{b} . I_{b}\right)$, as well as the main winding $\left(N_{c} . I_{c}\right)$. $\mathrm{R}_{\mathrm{gap}}$ is the reluctance of the air gap, and is considered constant. The components $R_{L}, R_{C}$, and $R_{R}$ are the reluctances of the left, center, and right arms respectively, and they are represented as a function of the permeability of the magnetic material $(\mu)$, since their values can vary depending on the operation point on the BH curve. Thus, for calculating these reluctances the referred model uses Brauer's equation, (1), which defines the BH characteristic curve of the magnetic material neglecting the hysteresis effect.

$$
H(B)=\left(k_{1} \cdot e^{k_{2} \cdot B^{2}}+k_{3}\right) \cdot B,
$$

where $k_{l}, k_{2}$, and $k_{3}$ are Brauer's model constants.

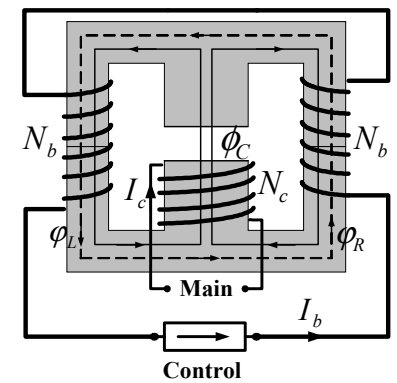

Fig. 1. Variable inductor based on double E-core structure.

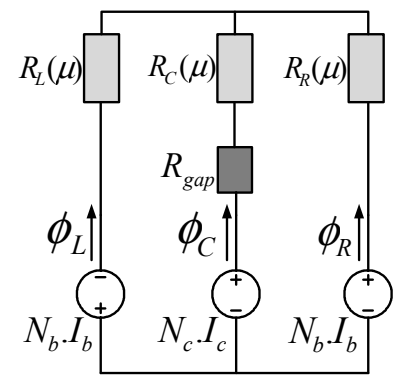

Fig. 2. Reluctance equivalent circuit of double E-core variable inductor shown in Fig. 1.

The afore-mentioned SPICE-based model has been replicated, specifically, in Simulink platform, to take advantage of the ability to integrate MATLAB script with Simulink library tools. This allows to include the device design calculations into the overall model of the system. Fig. 3 shows an example of inductance variation with the bias control current.

Two issues are related to this model; first, the hysteresis effect is not taken into consideration, second, the model input quantities are dependent on output ones which introduces difficulty in computations. In particular, this issue implies the necessity for implementing system calculation delays, with proper initialization, especially when including the device in a switching converter simulation. This furthermore complicates the simulation in different test platforms. Simulink, for instance, depends on mathematical modeling, thus the model causality must be decided [12].

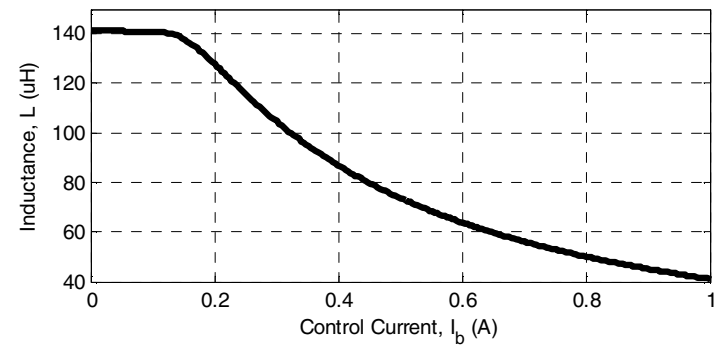

Fig. 3. Inductance variation with bias control current

In this paper the model of the variable inductor has been extended to include device losses, mainly core losses, AC winding losses, and Eddy currents. The following sections discuss the implementation and validation of the model.

\section{HYSTERESIS MODEL}

There are several methods to calculate the losses in a Ferromagnetic material, which were grouped by the authors in [13] to be three main approaches: hysteresis models, empirical equations, and loss separation. This paper undertakes the first approach, specifically the Jiles-Atherton (JA) hysteresis model [14], being the most suitable for development from a circuit simulation perspective [15].

\section{A. Model Implementation}

JA model separates the hysteresis function into reversible $\left(M_{r e v}\right)$ and irreversible magnetizations $\left(M_{i r r}\right)$, by computing these components along with the anhysteretic magnetization $\left(M_{a n}\right)$, based on Langevin function, the total magnetization $(M)$ can be reached. The model parameters are initially estimated by an iterative procedure to fit the model to the material $\mathrm{BH}$ curve data provided by the manufacturer. Fig. 4 shows a glance at the implementation of the JA equations [14]. For a given core size and magnetic material, the magnetic flux density $(B)$ can be estimated for a certain magnetic field intensity $(H)$ applied to the core.

\section{B. Test Setup for Measuring Core Losses}

To validate the implemented JA model, and study the effect of frequency on the model parameters, a test setup is developed to measure the core losses. There are mainly two approaches for measuring core losses [16]: electrical methods, and calorimeter methods. BH curve electrical measurement technique has been selected, specifically the two-winding method [17], since it is reported to be accurate for the frequency range under test in this study $(<100 \mathrm{kHz})[18]$. 


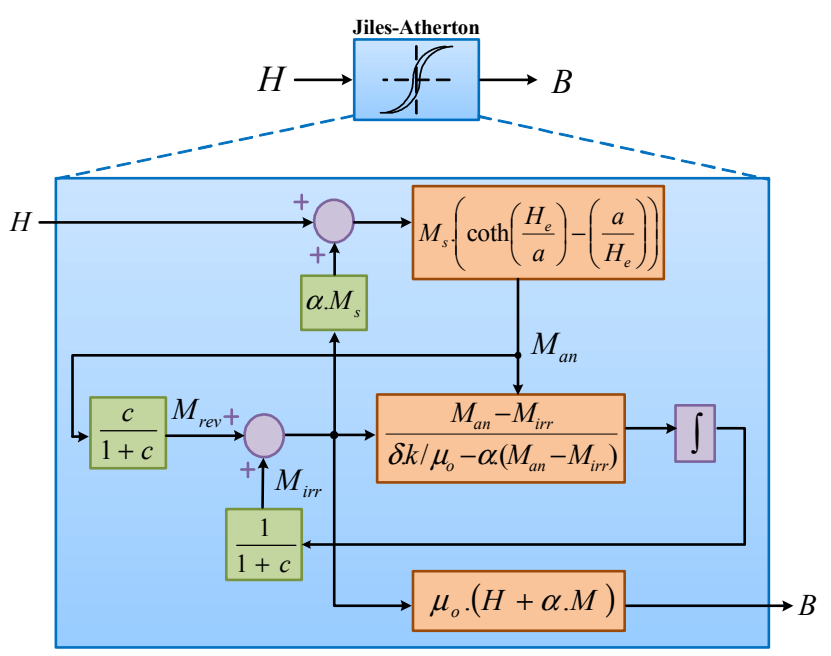

Fig. 4. Schematic of the implementation of JA model.

TABLE I. SPECIFICATIONS OF THE TEST SETUP

\begin{tabular}{|c|c|}
\hline \multicolumn{2}{|c|}{ Magnetic Core } \\
\hline Core material & N87 \\
\hline Core type and size & $\begin{array}{c}\text { Toroidal Core } \\
\text { R16.0 } \times 9.60 \times 6.30\end{array}$ \\
\hline Main winding no. of Turns (Np) & 5 turns \\
\hline Sensing winding No. of Turns (Ns) & 5 turns \\
\hline \multicolumn{2}{|l|}{ Test Setup } \\
\hline Input voltage & $30 \mathrm{~V}$ \\
\hline Maximum current & $3 \mathrm{~A}$ \\
\hline Frequency & $50 \mathrm{kHz}$ \\
\hline
\end{tabular}

The core used for the validation tests is a toroidal core with the design parameters listed in Table I. In addition to the main excitation winding $\left(N_{p}\right)$, a secondary sensing winding $\left(N_{s}\right)$ is added to sense the induced voltage due to the flux in the main one. The advantage of a separate winding is to exclude the voltage drop due to the resistance of the main winding. The magnetic flux density can thus be computed using (2).

$$
B=\frac{1}{N_{s} \cdot A} \cdot \int V_{s} \cdot d t
$$

where $A$ is the cross section area of the toroid, and $V_{s}$ is the opencircuit secondary winding voltage. The field intensity can thus be computed using (3).

$$
H=\frac{N_{p} \cdot I_{p}}{l},
$$

where $I_{p}$ is the current in the main winding, and $l$ is the effective length of the core.

Fig. 5 shows the experimental setup used for the measurement of core losses. The power stage used in the tests is a simple half-bridge converter which is preferable at low power levels. Also a square-waveform excitation voltage is sought for verifying the loss study under non-sinusoidal conditions. Fig. 6 depicts the open-circuit voltage measured at the secondary winding of the toroid, and the current flowing through the main winding. This plot aims to demonstrate the operation of the core in saturation, where the V/I magnitudes are the basis to use equations (2) and (3) in order to plot the measured $\mathrm{BH}$ curve. Fig. 7 illustrates this BH curve, obtained through measurements (solid line), compared with the simulated one obtained from JA model (dotted lines).

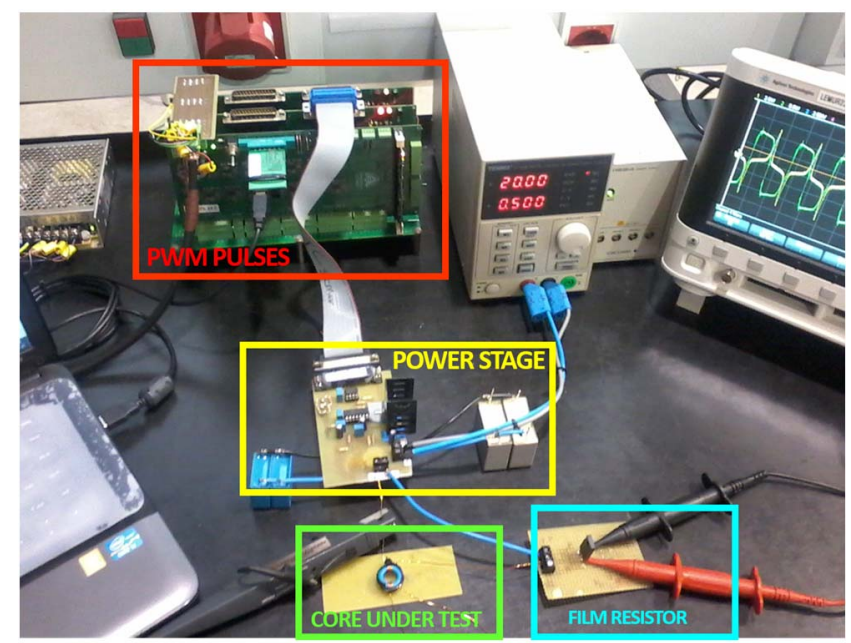

Fig. 5. Experimental setup used to measure BH curve.
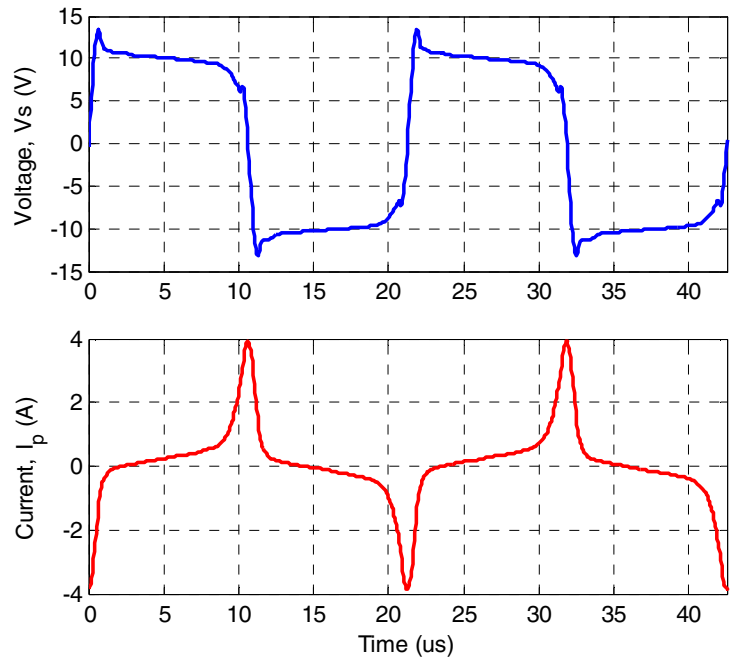

Fig. 6. Experimental measurements - The top plot illustrates the voltage applied to the toroid and the bottom plot illustrates the current through the main winding.

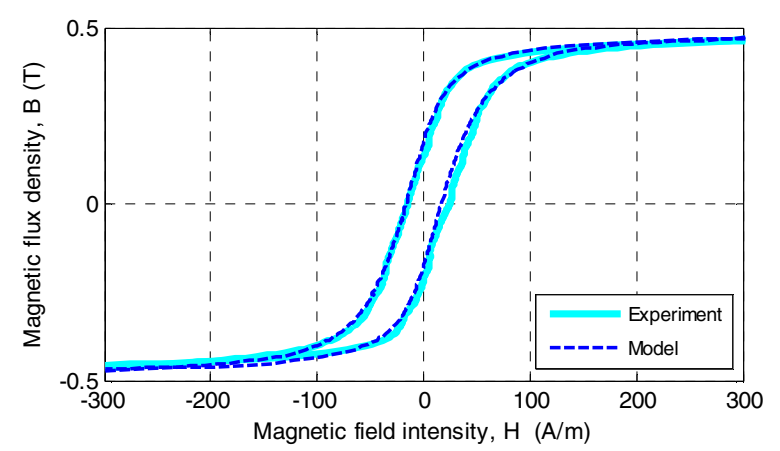

Fig. 7. BH curve based on JA model compared to experimental measurement - Model (dotted line) against experimental results (solid line). 


\section{Frequency Dependence of JA Parameters}

The shape of the BH curve changes as the frequency of the excitation voltage varies, and consequently the core losses are modified. This implies the variation of JA model parameters. It is thus of interest to develop a model that can be used for any frequency without having to readjust the parameters each time different operating conditions are considered. A practical approach has been taken by conducting a number of experiments to observe the variation of the JA parameters with frequency. Using curve fitting, expressions have been extracted for the parameters as a function of frequency. Fig. 8 shows the BH curve hysteresis loops, intending to demonstrate the comparison of the model (dotted lines) against experimental results (solid lines) for different frequency values. The results show that the model matches properly the obtained experimental measurements.

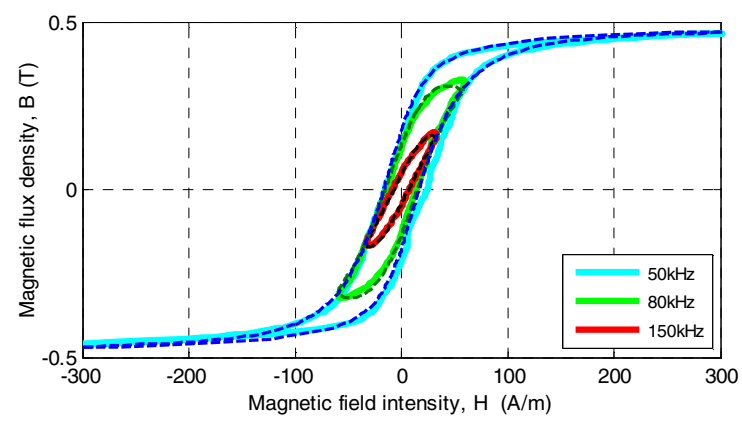

Fig. 8. BH curves for the prototype under different operation frequencies Model (dotted line) against experimental results (solid line).

\section{EDDY CURRENT LOSS MODEL}

In addition to core losses, especially as the frequency increases, Eddy current losses will significantly contribute to the device overall losses. Thus in order to compute the Eddy current losses, Dowell expression is used [19]. It is a simple method that provides fast computation of the equivalent winding resistance thus it can be easily integrated into the magnetic device model without extra complication. Although this is a great advantage still the expression in not valid for gapped cores [20], and this issue will be added as an improvement to this study. Fig. 9 illustrates the total winding impedance as a function of frequency to compare Dowell model against the experimental measurement obtained from an inductor implemented on an ETD49 core size (N87 material) with a 4-layer winding.

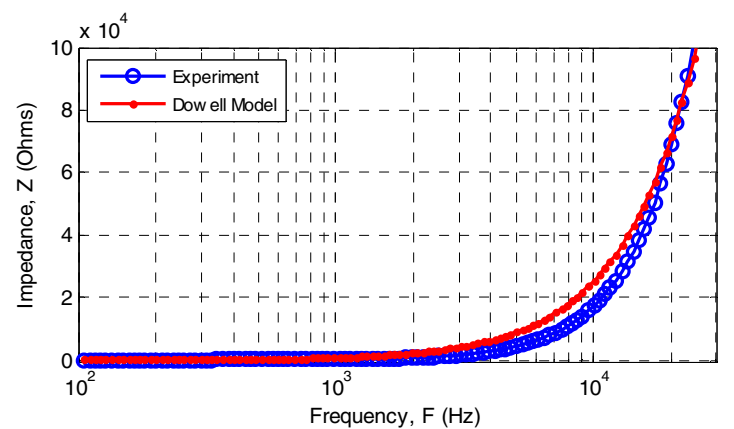

Fig. 9. Total winding impedance as a function of frequency - Model against experimental results.
The model shows an acceptable agreement with the measured winding impedance. Therefore, applying Dowell expressions, the total winding resistance can be computed as a function of the operation frequency, and added to the device model as a variable frequency-dependent resistor.

\section{APPLICATION OF LOSS MODELS TO IMPLEMENT THE VARIABLE INDUCTOR}

After verification of the loss models based on a simple toroidal structure, the study is applied to model the double Ecore variable inductor shown in Fig. 1. The model is implemented based on the reluctance circuit concept previously stated. However, the two issues associated with the previous reluctance model has been tackled. To avoid algebraic loops due to model causality, the magnetic core has been partitioned according to its operation as mentioned previously, thus defining each partition in terms of electrical inputs and outputs as follows.

The middle arm will have the main winding, and thus the input is the excitation voltage, while the output is expected to be the inductor current. The lateral arms will have the control windings, and thus the input is the control current, while the output is expected to be the induced voltage. Fig. 10a illustrates the magnetic part, which is the reluctance circuit of the device, and Fig. 10b illustrates the electrical part which is the windings' model, and is composed of the input voltage and the output current sources.

The reluctance circuit is composed of three branches. The left and right branches represent the magnetic circuits of the control arms of the device. The voltage source $\left(N_{b} . I_{b}\right)$ models the magnetomotive force created by each control winding. The voltage sources $\left(\phi_{R} R_{R}\right)$ and $\left(\phi_{L} R_{L}\right)$ model the variable reluctance of the magnetic path of the right and left arms respectively. Using the control current as the input quantity, the variable voltage sources' values are calculated based on the JA hysteresis model.

The middle branch represent the magnetic circuit of the main arm, the variable reluctance of the magnetic path is similarly represented by the voltage source $\left(\phi_{c} R_{c}\right)$, while in this case the magnetomotive force is the output quantity and is represented by a current source. The current in the main winding $\left(I_{c}\right)$ can thus be calculated by measuring the voltage across this current source and dividing by the number of turns of the winding $\left(N_{c}\right)$.

On the other hand, the electrical circuit, shown in Fig. 10b implements the electrical part of the three windings, which is represented by (4).

$$
V_{w}=N_{w} \cdot \frac{d \phi_{w}}{d t}
$$

where $V_{w}$ is the winding voltage, $\phi_{w}$ is the magnetic flux created by the winding, and $N_{w}$ is the number of turns of the winding. To account for winding losses, a constant resistor, $R_{d c}$, as well as a variable frequency-dependent resistor, $R_{a c}$, are added to the electrical circuit of the main arm. The resistors values accounts for the DC and AC winding resistances computed from Dowell model. 


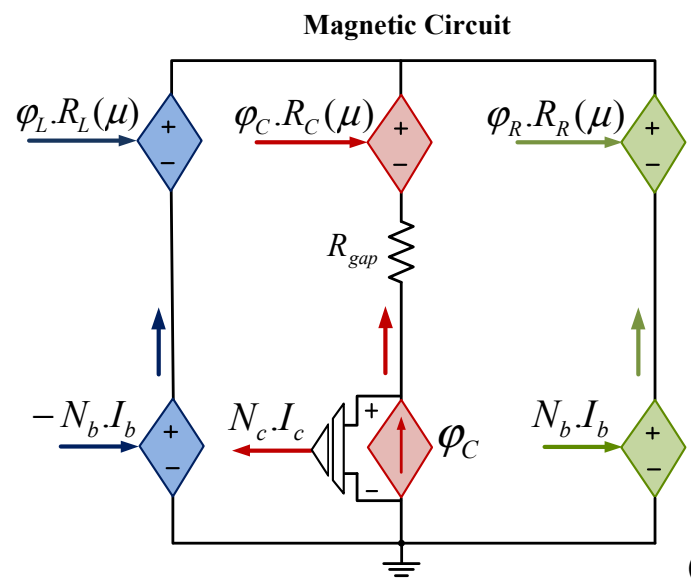

(a)

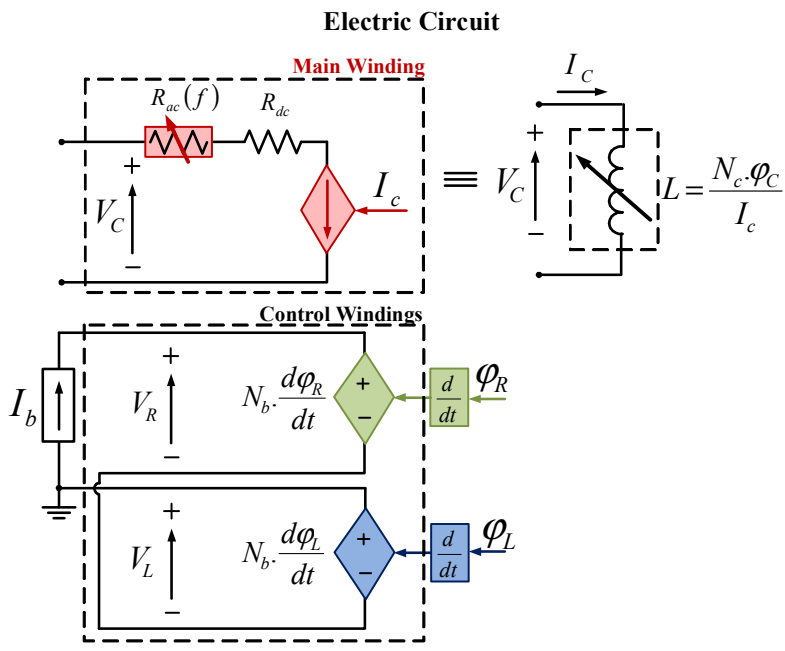

(b)

Fig. 10. Schematic of variable inductor model based on the reluctance circuit, (a) shows the magnetic circuit, and (b) shows the electric circuit.

\section{Model VALIDATION USING SimUlation AND EXPERIMENTAL RESULTS}

The simulations have been carried out to compare the proposed model, including hysteresis and Eddy current losses, with the replicated model that does not consider device losses. Those two simulation models have been compared against experimental measurements obtained from a variable inductor prototype, Fig. 11. The device has been developed based on the double E-core structure with the design specifications indicated in Table II, and the models have been adjusted correspondingly.

To validate the proposed model under small-signal analysis, the control windings of the device were connected to a variable DC voltage source, with a series resistor to provide a DC control current of maximum 1A. The control current was varied in steps from 0 to $1 \mathrm{~A}$, and the equivalent inductance seen from the main winding was measured using an impedance analyzer. Fig. 12 illustrates the equivalent inductance as a function of the bias control current obtained from both models compared against the experimental measurements. It can be observed that the proposed model that includes losses predicts the inductance within an acceptable range compared to the experimental one. On the other hand, the inductance predicted by the model that does not include losses shows a clear deviation from the experimental one as the control current increases.

The prototype has also been characterized under large-signal analysis. The test platform used to measure the BH curve is used to test the developed variable inductor. Similar to the smallsignal analysis, a DC control current is applied to the control windings and varied from 0 to $1 \mathrm{~A}$. However, in this case, the main winding is connected to the half-bridge converter, in order to apply a square voltage waveform to the inductor. The inductance is thus calculated from the RMS values of the applied voltage and inductor main winding current. Similar to the previous analysis, Fig. 13 illustrates the equivalent inductance as a function of the bias control current. As the control current increases towards $1 \mathrm{~A}$, the inductance predicted by the proposed model is observed to approach the experimental measurement. On the contrary, the inductance predicted by the model that does not include losses deviates apart from the experimental one.

Finally, the inductor RMS current has been measured and compared to the one predicted by the proposed model including device losses. Fig. 14 shows the comparison results, as it can be observed the model can predict the variation of inductor current as a function of the bias control current with acceptable accuracy.

TABLE II. SPECIFICATIONS OF THE VARIABLE INDUCTOR

\begin{tabular}{|c|c|}
\hline Core material & N87 \\
\hline Core type and size & ETD core \\
& ETD49/25/16 \\
\hline Main winding No. of Turns & 23 turns \\
\hline Control winding No. of Turns & 55 turns \\
\hline
\end{tabular}

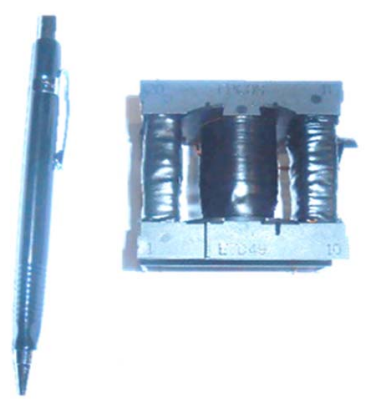

Fig. 11. Variable inductor prototype based on double E-core structure.

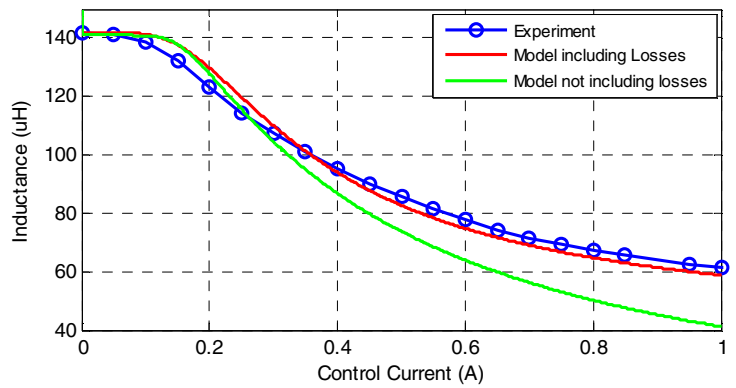

Fig. 12. Small-signal characterization of the variable inductor prototype comparing simulation models with experimental results. 


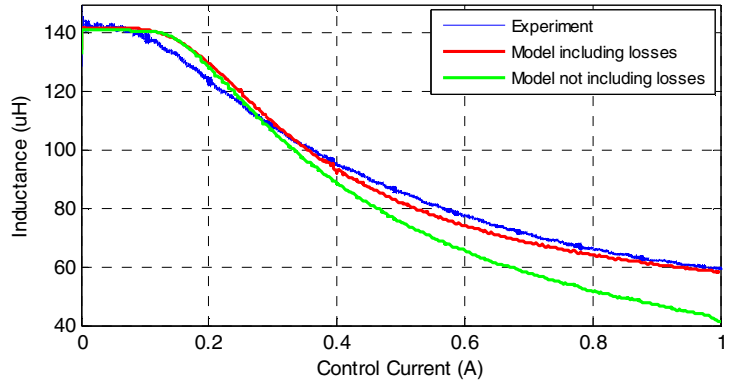

Fig. 13. Large-signal characterization of the variable inductor prototype comparing simulation models with experimental results.

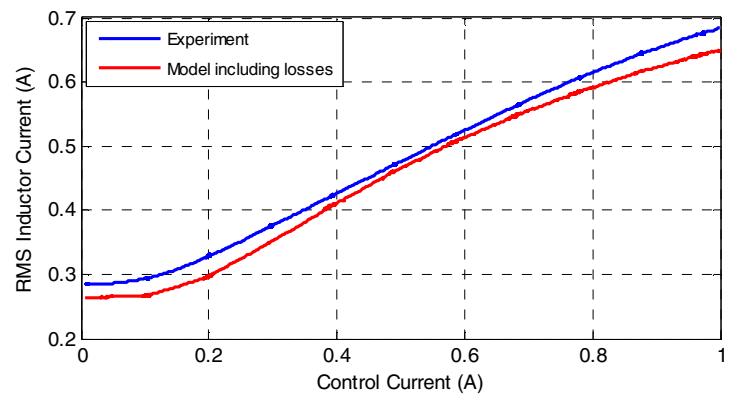

Fig. 14. Comparison between experimental and model results - RMS inductor current as a function of bias control current.

\section{CONCLUSIONS}

A model has been developed, which can predict the inductance variation as a function of the control current in a controlled magnetic regulator. The main contribution of this work is that the model, developed to be used in several simulation platforms, takes into consideration hysteresis as well as Eddy current losses in the device. A double E-core variable inductor prototype has been characterized under small-signal as well as large-signal analysis to assess the accuracy of the model. The proposed approach is considered an autonomous tool that can analyze any given set of data (simulated or experimental) for a magnetic core, detect the operation frequency, and correspondingly, adjust the magnetic core model with the hysteresis parameters as well as the Eddy current losses, and finally predict the inductance as a function of the control current, along with other electric and magnetic quantities that characterize the magnetic core operation. Therefore, using only one simulator environment, the design parameters for the device, as well as the analysis of the whole system is provided.

\section{REFERENCES}

[1] Takach, Margarita D., and Peter O. Lauritzen. "Survey of magnetic core models." Applied Power Electronics Conference and Exposition, 1995. APEC'95. Conference Proceedings 1995., Tenth Annual. Vol. 2. IEEE, 1995.

[2] M. S. Perdigão, "Research and development on new control techniques for electronic ballasts based on magnetic regulators," Ph.D. dissertation, Dept. Elect. Comput. Eng., Univ. Coimbra, Coimbra, Portugal, Jun., 2012.
[3] Qianhong Chen; Ligang Xu; Xinbo Ruan; Siu Chung Wong; Tse, C.K.; "Gyrator-Capacitor Simulation Model of Nonlinear Magnetic Core," Applied Power Electronics Conference and Exposition, 2009. APEC 2009. Twenty-Fourth Annual IEEE, vol., no., pp.1740-1746, 15-19 Feb. 2009.

[4] Ludwig, G.W.; El-Hamamsy, S.-A.; "Coupled inductance and reluctance models of magnetic components," Power Electronics, IEEE Transactions on, vol.6, no.2, pp.240-250, Apr 1991.

[5] J. M. Alonso, G. Martínez, M. Perdigão, M. Cosetin and R. N. do Prado, "Modeling magnetic devices using SPICE: Application to variable inductors," 2016 IEEE Applied Power Electronics Conference and Exposition (APEC), Long Beach, CA, 2016, pp. 1115-1122.

[6] MANDACHE, Lucian, Dumitru TOPAN, and Ioana Gabriela Sîrbu. "Accurate time-domain simulation of nonlinear inductors including hysteresis and eddy-current effects." Proceedings of the World Congress on Engineering. Vol. 2. 2011.

[7] H. Fan and H. Li, "High-Frequency Transformer Isolated Bidirectional DC-DC Converter Modules With High Efficiency Over Wide Load Range for $20 \mathrm{kVA}$ Solid-State Transformer," in IEEE Transactions on Power Electronics, vol. 26, no. 12, pp. 3599-3608, Dec. 2011.

[8] Burgio, A.; Menniti, D.; Motta, M.; Pinnarelli, A.; Sorrentino, N.; Vizza, P., "A laboratory model of a dual active bridge DC-DC converter for a smart user network," in Environment and Electrical Engineering (EEEIC), 2015 IEEE 15th International Conference on , vol., no., pp.997-1002, 1013 June 2015.

[9] PSIM User's Guide: https://www.myway.co.jp/products/psim/dlfiles/pdf/PSIM_User_Manual V9.0.2.pdf

[10] Simulink Documentation: https://es.mathworks.com/help/simulink/index.html?s_cid=doc_ftr

[11] D. Medini and S. Ben-Yaakov, "A current-controlled variable inductor for high frequency resonant power circuits," in Proc. IEEE APEC, 1994, pp. 219-225.

[12] Johansson, P. and Andersson, B. (2008) Comparison of Simulation Programs for Supercapacitor Modelling. Göteborg : Chalmers University of Technology.

[13] J. Reinert, A. Brockmeyer and R. W. A. A. De Doncker, "Calculation of losses in ferro- and ferrimagnetic materials based on the modified Steinmetz equation," in IEEE Transactions on Industry Applications, vol. 37, no. 4, pp. 1055-1061, Jul/Aug 2001.

[14] D.C. JILES \& D.L. ATHERTON, "Ferromagnetic Hysteresis", IEEE Transactions on Magnetics, Vol 19, No. 5, Sep 1983, pp2183-2185.

[15] Peter Reid Wilson, "Modelling and Simulation of Magnetic Components in Electric Circuits," Ph.D. dissertation, Dept. Elect. Comput. Eng., Univ. Southampton, Nov., 2001.

[16] C. Xiao, G. Chen and W. G. H. Odendaal, "Overview of Power Loss Measurement Techniques in Power Electronics Systems," in IEEE Transactions on Industry Applications, vol. 43, no. 3, pp. 657-664, Mayjune 2007.

[17] V. J. Thottuvelil, T. G. Wilson and H. A. Owen, "High-frequency measurement techniques for magnetic cores," in IEEE Transactions on Power Electronics, vol. 5, no. 1, pp. 41-53, Jan 1990.

[18] M. Mu, Q. Li, D. J. Gilham, F. C. Lee and K. D. T. Ngo, "New Core Loss Measurement Method for High-Frequency Magnetic Materials," in IEEE Transactions on Power Electronics, vol. 29, no. 8, pp. 4374-4381, Aug. 2014.

[19] P. L. Dowell, "Effects of eddy currents in transformer windings," in Electrical Engineers, Proceedings of the Institution of, vol. 113, no. 8, pp. 1387-1394, August 1966.

[20] F. A. Holguín, R. Asensi, R. Prieto and J. A. Cobos, "Simple analytical approach for the calculation of winding resistance in gapped magnetic components," 2014 IEEE Applied Power Electronics Conference and Exposition - APEC 2014, Fort Worth, TX, 2014, pp. 2609-2614. 